\title{
Historical Note: The Evolution of Cortical Bone Trajectory and Associated Techniques
}

Sihyong J. Kim ${ }^{1,3}$; Ralph J. Mobbs ${ }^{1,2}$; Pragadesh Natarajan ${ }^{1,2}$; R. Dineth Fonseka1,2; William

$$
\text { R. Walsh }{ }^{3}
$$

${ }^{1}$ NeuroSpine Surgery Research Group, Sydney, Australia

${ }^{2}$ NeuroSpine Clinic, Prince of Wales Private Hospital, Sydney, Australia

${ }^{3}$ Surgical and Orthopaedics Research Lab, Prince of Wales Hospital, Sydney, Australia

Running Title:

History of Cortical Bone Trajectory

Corresponding Author:

Sihyong J. Kim

NeuroSpine Surgery Research Group

Suite 7, Level 7 Prince of Wales Private Hospital

Barker St, Randwick,

New South Wales, 2031, Australia

Tel: 0452390105

Email: usujake@gmail.com

\section{Conflicts of Interest:}

The authors declare that there no conflicts of interest.

\section{Authors' Contribution:}

S.J. Kim was the principal author, responsible for conducting the literature review and writing the primary manuscript. R.J. Mobbs provided the direction for the paper, provided a clinical background to CBT and the figures displayed in this manuscript, and revised the first 
manuscript. P. Natarajan and R.D. Fonseka assisted with data/literature collection and provided revisions of the final manuscript. W.R. Walsh provided biomechanical background to the CBT to the primary author and reviewed the final manuscript.

\section{Ethics Approval}

No ethics approval was not required as this was a historical review paper and did not require

(1) biomechanical testing using cadaveric/animal specimen or (2) any clinical participants. 


\begin{abstract}
Cortical bone trajectory (CBT) for posterior fixation with pedicle screws is considered a relatively new alternative trajectory that travels in the medio-lateral direction in the transverse plane and in the caudo-cephalad path in the sagittal plane. Various biomechanical studies have already validated its superior pullout strength and mechanical stability over the traditional trajectory of convergent pedicle screws. Due to the relatively medial starting point of this trajectory, the CBT also poses the clinical advantage of requiring a smaller surgical field of exposure, thus minimizing tissue and muscle injury while reducing operative time and intraoperative blood loss. The evolution of CBT through time has closely been linked to the unwavering philosophy of prioritizing patient outcomes, advancements in neuronavigational technology, and the mounting biomechanical, morphometric, and clinical evidence. In this historical review, we provide a unique perspective on how CBT surgical technique has developed through time, highlighting key milestones and attempting to explain its explosive rise in popularity.
\end{abstract}

\title{
KEYWORDS
}

Cortical Bone Trajectory, Pedicle Screws, Spinal Surgery, Historical Review 


\section{INTRODUCTION}

From its first conception by Roy-Camille in 1963, the use of pedicle screw fixation to provide immediate stability of the spine without compromising the mobility of additional spinal segments has gained widespread acceptance and popularity over the past few decades ${ }^{1,}$

2. Since then, pedicle screw fixation has been considered the mainstay of spinal fusion, boasting superior biomechanical advantages and successful fusion rates over other methods of fixation such as wires, bands, and hooks ${ }^{2-5}$. The traditional trajectory for pedicle screws has been described to be transpedicular in nature, following the latero-medial anatomical axis of the pedicle and directed cephalo-caudally in the sagittal plane. However, to achieve this convergent "triangulation" of screws from the lateral starting point, significant tissue dissection and muscle retraction are necessary, which can only cause muscle trauma and extend recovery time (Figure 1). Furthermore, negative complications can occur with traditional trajectory such as screw loosening, pullout, or breakage leading to surgical construct failure as the screws embed in the less dense cancellous bone of the pedicles. Early strategies to mitigate the effects of screw loosening in the context of low bone mineral density included altering screw properties such as its diameter or through adjuvant use of cement through fenestrated or cannulated screws ${ }^{6,7}$. However, larger screws have been determined to pose greater risk of encroachment onto the delicate surrounding neurovascular structures, whereas the use of cement has been associated with screw breakages and cement leakage into the vertebral canal, which, in turn, can result in disastrous neurological deficits 6 , 8,9 .

Recently, an alternative trajectory investigated and published by Santoni et al. in 2009 has been gaining increasing attention ${ }^{10}$. This trajectory follows a medio-lateral direction in the transverse plane and a caudo-cephalad path in the sagittal plane, gaining predominantly 
cortical bone purchase and hence was coined the "cortical bone trajectory (CBT)." ${ }^{10}$ Due to the medial starting point of this "novel" trajectory and the fact that the lateral aspect of the pars does not need to be exposed, this technique has offered an immediate advantage of requiring a smaller incision and significantly less muscle dissection, thus reducing muscle injury over the traditional trajectory. Furthermore, increased cortical bone purchase with this trajectory has been observed to significantly improve vital biomechanical parameters such as pullout strength and mechanical stability. Consequently, it is of no surprise that the CBT has become a topic of interest among spinal surgeons, especially in the often challenging management of osteoporotic and osteopenic patients. Thus, this report aims to elucidate the historical development of this novel trajectory from its early conception and further shed light on the recent revolutions made in this field. 


\section{HISTORICAL OVERVIEW}

\section{First conceptualization}

It can be argued that the earliest conceptualization of the modern CBT was described by Buck in the 1970s as a new approach to repairing spondylolisthesis secondary to a pars interarticularis defect ${ }^{11}$. Buck has described the trajectory of a lag screw that travels "upwards, forwards, and slightly outwards," crossing the pars interarticularis defect and ensuring that the threads of the screw gain enough purchase on the posterior and anterior cortical plates of the lamina and on the cortical bone of the pedicles ${ }^{11}$. As per application of this technique on 16 original patients, it revealed a success rate of $94 \%$, with 1 case of failure and 2 complications, which was a significant improvement from previous methods for surgical fusion which reported only $65 \%-75 \%$ of operations with satisfactory results ${ }^{12}$. Despite the novelty and apparent success of this maneuver, Buck's technique has failed to attract significant interest in the surgical community throughout the twentieth century, perhaps due to its introduction as a highly specialized indication for pars interarticularis defect exclusively. Alternatively, the notion that this technique was limited to spondylolisthesis due to pars defect cases with gaps measuring less than $4 \mathrm{~mm}$ may have further curtailed its popularity.

Indeed, in the years following Buck's conception of bilateral screw trajectory, other techniques for internal vertebral fixation have also emerged, such as the use of cerclage wires as proposed by Scott and published by Bradford (1976) and the securing of hooks underneath the lamina as conceptualized by Morscher $(1984)^{13-15}$. However, a comparative meta-analysis of Buck's, Scott's, and Morscher's technique has revealed that Buck's technique had the highest rate of fusion (83.53\%), followed by Scott's (81.57\%) and finally Morcher's $(77.72 \%)^{16}$ 


\section{Convergent pedicle angles become cemented as the norm}

By the late 1990s, pedicle screw fixation systems have become widely available, after having been subjected to numerous design alterations since the initial conception of the pedicle screws by Roy-Camille in $1963^{1,2}$. Some of the most common issues surrounding the efficacy of the pedicle screws were loosening and screw breaking, which have led several biomechanical studies to examine factors that evaluate pedicle screw stability ${ }^{17}, 18$. An important consideration for pedicle screws was the insertion angle or the intended trajectory of the screws as it passes through the posterior elements. Stemming from the morphometric analyses of pedicles that "mapped" its transverse angles, studies such as those conducted by Barber et al. (1998) found that pedicle screw trajectories that converge from a lateral starting point at an angle of approximately $30^{\circ}$, thus following the anatomical axis of the pedicles, offered more resistance to axial pullout and withstood higher cyclical loads at the clinical threshold of screw loosening compared to (parallel) pedicle screws at a convergent angle of $0^{\circ 19,20}$. Furthermore, Cook et al. (2000) performed a combined biomechanical and clinical study on expansive screws to propose that axial pullout strength has increased with greater angular distance from the midline ${ }^{21}$. Later, a comparison of pedicle screw trajectories following the anatomical axes of the pedicle (anatomic trajectory) with straight-forward (follows the pedicle axis in the axial plane and parallel to the superior endplate in the sagittal plane) and straight-ahead (parallels the midsagittal line in the axial plane and the superior endplates in the sagittal plane) pedicle trajectories by Dhawan et al. (2008) found that the former trajectory demonstrated the maximum effective pedicle diameter and insertional $\operatorname{arc}^{22}$. Ultimately, findings of studies such as these contributed to the justification of the traditional trajectory today. 


\section{Re-invigorated attempts at alternative trajectories}

Despite the mounting evidence supporting the latero-medial trajectory (convergent pedicle screws) of pedicle screws, several attempts at an alternate medio-lateral and craniocaudal pedicular trajectory, resembling Buck's original technique, were made in the early 2000s. Steel and Mobbs et al. (2004) described a similar pedicular trajectory for the management of 18 patients who were suffering from traumatic thoraco-lumbar burst fractures between the years 2001 and 2003, with 17 out of these patients reporting successful fusions with less back pain, or limitation of function 3 months post-operatively ${ }^{23}$. The authors described the procedure as the medio-latero-superior trajectory (MLST, Figure 2), entering from the medial aspect of the pars ${ }^{23}$. The perceived benefit of this technique was its minimally invasive nature, as it requires only a smaller surgical field of exposure, which, in turn, reduced incision length, muscle dissection, and surgical trauma ${ }^{23,24}$. Over the next 10 years, the authors expanded this technique in treating other degenerative conditions and nonspondylotic tumors beyond the original indications of trauma ${ }^{24}$.

\section{The catalyst for CBT validation}

With the aging population, a growing number of people receiving pedicle screw fixation consist mostly of elderly patients, who are often suffering from many comorbidities such as osteoporosis and osteopenia. Indeed, it has been estimated that by 2022, 6.2 million Australians over the age of 50 will have either osteoporosis or osteopenia, that is, a $31 \%$ increase from $2012^{25}$. As a result, studies investigating the relationship between bone mineral density and biomechanical parameters of pedicle screw fixation such as pullout strength have gradually increased from the 1990s. These studies found that pedicle fixation has become increasingly difficult in the vertebrae of low bone mineral density, predisposing the patient to serious complications such as screw loosening or pullout, device migration, or breakage ${ }^{26-29}$. 
A quantitative computed tomography (CT) study of bone mineral density of the pedicles by Hirano et al. (1997) revealed that due to the thinner than normal cortex in conjunction with lower bone mineral density in the subcortical bone, increasing screw diameter did not enhance screw stability and may even lead to undesired fracturing of the thinned pedicle cortex $^{30}$. However, the search for a solution to fixation in osteoporotic bone did not stop there. Several studies have commented on the enhanced pullout strength of techniques using pedicles augmented with bone cement. For example, Cook et al. (2004) reported a greater than twofold increase in pullout strength with polymethyl methacrylate (PMMA) cemented expandable screw compared with a non-cemented expandable screw in severely osteoporotic bone ${ }^{26}$. Furthermore, Yazu et al. (2005) performed axial pullout testing on a fenestrated pedicle screw augmented with calcium phosphate cement, which revealed a pullout strength of $637 \mathrm{~N}$ compared to the standard $258 \mathrm{~N}$ of an average screw ${ }^{7}$. While these results sounded promising, cement augmentation was deemed not ideal as it led to fractures at the bone screw interface or cement leakage into the vertebral canal leading to serious neurological damage ${ }^{7,8}$, ${ }^{26}$. Additionally, rates of cement leakage are seen to increase with higher dosages and with the tip of the screw "converging" on the midline of the vertebral body such as in the traditional trajectory ${ }^{31}$. Furthermore, screws that are augmented with cement could not be revised in the events of mispositioning or complicated surgeries.

In the midst of the search for the solution to osteoporosis dilemma, Santoni et al. (2009) re-visited an alternative pedicle screw trajectory described initially by Buck and carried out by some surgeons prior to $2009^{10}$. Santoni et al. described this trajectory to take advantage of only the cortical bone of the vertebra as it travels into the medio-lateral direction in the transverse plane and in the caudo-cephalad pathway in the sagittal plane ${ }^{10}$. This was a theoretically ideal solution to the osteoporosis problem as cortical bone density is 
less affected by the condition than the cancellous bone of the vertebra ${ }^{32}$. As expected, Santoni et al. demonstrated that the "CBT" was juxtaposed by significantly higher bone density compared to traditional trajectories as seen on qCT scans ${ }^{10}$. Consequently, the mean pullout strength for the CBT was approximately $367 \mathrm{~N}$ compared to $288 \mathrm{~N}$ of a traditional trajectory, equating to about a $30 \%$ improvement in pullout resistance and stability for the CBT screws ${ }^{10}$.

The findings of Santoni et al. have followed an important biomechanical study by Lehman et al. (2003) and Sterba et al. (2007) which found that, contrary to previous studies, screws inserted parallel and angled upward, without convergence, had greater pullout strength and better fatigue performance than convergent pedicle screws ${ }^{33,34}$. Furthermore, this study suggested the practical benefits of non-convergent screws as it did not require extensive dissection and retraction of the paraspinal musculature and thus could be beneficial in the demographic populations of larger body habitus or those requiring a minimally invasive approach $^{33}$. These studies served as the fulcrum upon which an alternative trajectory, namely, the CBT, could finally be balanced and evaluated in comparison with the traditional and already widely accepted trajectory.

\section{Mounting evidence for $C B T$}

CBT has become widely recognized after the seminal work by Santoni et al., which led to several biomechanical, morphometric, and some clinical studies that aimed to compare CBT with traditional trajectories. This was a significant paradigm shift for the literature on CBT as prior to this, the traditional trajectory was only compared with straight/parallel screws, showing mixed findings in the early 2000s. It can be argued that the academic excitement for CBT began with Matsukawa et al.'s first of the many studies that used the CT scan of 100 adults to quantitatively describe CBT parameters in terms of diameter, length, and lateral and cephalad angles ${ }^{35}$. This allowed the original MLST/CBT technique to be 
optimized with a more superior starting point, shallower cephalad angle, and a longer pedicle insertion length which was reported to possess both morphological and biomechanical advantages in terms of cortical bone purchase (Figure 3).

Further efforts were made by Matsukawa et al. to expand on the available biomechanical and morphometric evidence for CBT. Of particular importance in the historical development of CBT was Matsukawa et al.’s investigation on insertional torque. Previously, in 1993, a human cadaveric study performed by Zdeblick et al. found that insertional torque has positively correlated with pullout strength ${ }^{36}$. This served as the overarching premise for Matsukawa et al.'s study in 2014 that aimed to evaluate the comparative stability of CBT and traditional trajectory using insertional torque as an objective parameter ${ }^{32}$. Mean insertional torque for traditional pedicle screws replicated biomechanical data on insertional torque conducted in the past ${ }^{37}$. Interestingly, direct comparison of $\mathrm{CBT}$ with the traditional trajectory found that the mean maximum insertional torque of CBT was higher by 1.7-fold, and, for the CBT trajectory, insertional torque gradually increased as the screw continued to purchase more cortical bone with increasing insertion depth, whereas the insertional torque plateaued earlier for the traditional trajectory as it delved further into cancellous bone ${ }^{32}$. A more recent morphometric analysis conducted by Matsukawa et al. examining CT scans of 50 adults with low thoracic CBT compared the insertional torque of CBT to traditional screw placement in 24 cadaveric thoracic vertebrae and found that, on average, CBT demonstrated a $53.8 \%$ higher torque ${ }^{38}$

Other biomechanical studies provided additional support for and against CBT. For example, Perez-Orribo et al. (2013)'s biomechanical analysis of cadaveric lumbar specimen revealed that there were no statistically significant differences in terms of stability between 
CBT and traditional pedicle screws ${ }^{39}$. Furthermore, it was found that screws using the traditional trajectory were stiffer during axial rotation bringing into question the concern for increased micromotion at the screw bone interface with CBT, potentially increasing the risk of screw loosening ${ }^{39}$. Conversely, a study performed by Calvert et al. in 2014 found that both CBT and traditional screws produced similar stiffness in axial rotation as well as flexion, extension, and lateral bending in salvage cases of failed pedicle screw constructs, suggesting that CBT would be a viable alternative in these $\operatorname{cases}^{40}$.

A major point of contention regarding the mounting evidence for and against $\mathrm{CBT}$ is in relation to the reliability of biomechanical studies that test both the safety/longevity and efficacy of these fixation systems. Historically, pullout strength has been the mainstay in biomechanical testing and has arguably contributed to the productive discussions regarding pedicle insertion techniques and trajectories that propagated the development of CBT. However, it is important to note that tests such as axial pullout are conducted in vitro and, therefore, do not replicate the biological and physiological loads a normal spine or spinal fixation system will undergo in vivo. In order for the CBT to be further recognized, accurate in vivo evidence that supports its superiority over traditional trajectories are required in conjunction with clinical studies that could elucidate the real-life applicability of this technique. In such a way, a human cadaveric biomechanical study by Baluch et al. was critical in demonstrating the superior resistance of the CBT to craniocaudal toggling loads that aimed to model the natural forces that cause screw pullout and loosening in physiological flexion/extension, lateral bending, and axial rotation ${ }^{41}$.

Despite a lack of clinical data in the literature since 2004 , to the best of our knowledge, a variety of clinical applications of CBT emerged from 2013 onward, which are 
seemingly concordant with the laboratory studies of Matsukawa et al. and others ${ }^{42,43}$. A complete correction of degenerative lumbar scoliosis using CBT screws in a patient with osteoporosis was presented by Ueno et al. in a 2013 case report ${ }^{44}$. Fewer rates of complications such as screw loosening and pseudoarthrosis were found for CBT screws compared to screws inserted using the traditional trajectory in a 2014 retrospective study conducted by Gonchar et al. comparing 100 CBT screws with 63 traditional trajectory screws $^{45}$. Interestingly, they reported lower operation times as well as intraoperative blood loss (177 $\mathrm{ml}$ for CBT and $334 \mathrm{ml}$ for traditional) for $\mathrm{CBT}^{45}$. No complications were reported by Iwatsuki et al. in 8 cases of isthmus-guided CBT screw fixation for patients with lumbar degenerative spondylolisthesis ${ }^{46}$. Similarly, Mizuno et al. reported 12 cases of single-level lumbar spondylolisthesis using a posterior or transforaminal lumbar interbody fusion with $\mathrm{CBT}^{47}$. However, a significant finding was that four screws were deemed to have perforated the walls of the pedicles and vertebral bodies ${ }^{47}$. A comparison of 16 cases of CBT with 19 open posterior lumbar interbody fusion by Okudaira et al. reported shorter operation times (148 minutes for CBT and 184 minutes for traditional), lesser blood loss (132 $\mathrm{g}$ for CBT and $184 \mathrm{~g}$ for traditional), and fewer days needed to return to normal temperature (4.6 days for CBT and 7.8 days for traditional $)^{48}$. A unique hybrid contralateral CBT and traditional screw placement using CT-guided navigation was employed by Rodriguez et al. for symptomatic adjacent-segment lumbar disease ${ }^{49}$.

\section{Evolution of technique and directions for the future}

From the first conceptualization of the trajectory by Buck in the 1970s for pars repair, then some reports in the early 2000s, to the first formal description of the modern CBT by Santoni et al. in 2009 to improvements to CBT galvanized by the biomechanical, morphometric, and clinical analyses made by Matsukawa et al. and others from 2013 onward, 
CBT has indeed become a potent contender to the traditional trajectory for pedicle insertion. In the last 5 years, the literature has been a hub for interesting advancements in the nuances of CBT and sometimes an arena for continuing the contentious debate between the two trajectories. For example, in 2018, Xuan et al. published a radiographic assessment of CBT for $\mathrm{T} 1-\mathrm{T} 8$, recommending a specific screw dimension of $4.5 \times 25.0-30.0 \mathrm{~mm}$ in this spinal region by evaluating incidences of pedicle wall penetration or violation of $160 \mathrm{CBT}$ screws $^{50}$. Possibilities of expanding the indications of CBT to a wider array of cases are emerging with Lai et al., as they have compared the clinical effect of CBT in senile patients with lumbar tuberculosis in $2020^{51}$. As expected, CBT was advantageous over traditional screw trajectory in that it produced less trauma while maintaining stronger screw holding force ${ }^{51}$.

Indeed, the role of CBT has extended beyond the conventional spinal segments of the thoracic and lumbar spine with recent reports of CBT being utilized for lumbosacral fixation. Lumbosacral fixation with long-segment fusion constructs to the sacrum can be an indication for spinal pathologies such as osteoporosis, fractures, infections, and tumors ${ }^{52}$. However, surgery to this area can be quite demanding and challenging owing to its complex anatomy, proximity to surrounding visceral structures, poor bone quality, and significant mechanical forces, thus leading to undesirable outcomes such as pseudoarthrosis, hardware failure, and loss of correction ${ }^{53}$. In 2020, Matsukawa et al. provided an interesting development in the CBT technique by combining it with the sacral-alar-iliac screw technique, producing a minimally invasive approach to sacral fixation. The sacral CBT trajectory was described to start more medial compared to conventional approaches and was directed anteriorly without convergence, penetrating the middle of the sacral endplate, thus engaging in dense sacral bone ${ }^{52}$. The authors discussed that the main advantages of this technique were as follows: (1) it allowed for ease of rod insertion, (2) it minimized the risk of rod fracture, (3) it required 
less lateral muscle dissection and thus decreased the risk of ischemic necrosis and denervation of posterior muscles, and 4) it had higher cortical purchase that resulted in superior biomechanical performance of this technique over conventional sacral fixation screws $^{52}$.

Recently, significant interest has been garnered over the use of robots for spinal surgery in order to achieve an even more minimally invasive procedure. These robot-assisted procedures aim to further reduce the incidence of undesirable outcomes such as neurovascular encroachment and neurological deficits with CBT applications. A study by Le et al. compared standard CBT with robot-assisted CBT using a navigational orthopedic robot and found that robot-assisted surgery was able to reduce the rate of facet joint violation significantly from $41.3 \%$ in CBT to $17.3 \%$ in the robot group ${ }^{54}$. Furthermore, robot-assisted surgeries are becoming alternatives to traditional surgical navigation and trajectory planning using intraoperative CT or fluoroscopy. Furthermore, a retrospective study by Khan et al., which compared 22 patients who underwent robot-assisted CBT to 18 patients who underwent CT-navigated CBT, found better accuracy (100\%) for robot-guided CBTs ${ }^{55}$. While this study was limited due to its small sample size that curtails the generalizability of its findings, it represents the beginning of a new era of history for CBT and pedicle screw fixation in general, where one may raise the question of how much further these techniques can be enhanced in terms of safety and efficacy through the aid of robotics and machine learning.

Another interesting point of discussion for modern CBT revolves around the concept of 3Dprinted patient-specific guides. Personalized or precision medicine is a relatively modern concept that relies on innovations in engineering, genetics, data science, and so on to achieve 
a greater level of patient-centered and individualistic care ${ }^{56}$. Kim et al. demonstrated the use of 3D printing to produce patient-specific drill guides for a posterior lumbar interbody fusion with CBT of a 71-year-old woman presenting with spinal canal stenosis and grade 2 mobile spondylolisthesis at L4-L5 ${ }^{57}$. The authors reported benefits of avoiding the high start-up and maintenance cost for CT neuronavigation. Furthermore, the 3D-printed models of the patient's spinal segments have significantly helped in pre-operative planning, thus minimizing operative time. Consequently, extended periods of anesthesia and rates of infections were greatly reduced ${ }^{57,58}$. Another retrospective analysis of 30 patients by Maruo et al. found that the use of 3D-printed guides for CBT by a surgeon "inexperienced" in the CBT technique increased the accuracy of CBT screw placement to $97 \%$ from $91 \%$. Furthermore, similar technique used by Marengo et al. suggested that 3D-printed guides may reduce the incidence of nerve damage ${ }^{60}$. While further clinical data and investigations are necessary to test the safety and efficacy of 3D-printed guides and other such adjuncts to CBT, these studies are suggestive of the potential future for CBT and spinal surgery in general, where advancements in patient outcome are propagated by combining medicine with industry innovations.

\section{CONCLUSION}

CBT is a recent advancement in one of the many techniques in spinal fixation surgery. Its philosophy is rooted in prioritizing patient outcome and minimizing negative complications such as tissue and muscle damage, in addition to potential neurovascular injury. The historical development of CBT offers a unique perspective into the development and evolution of a surgical technique, influenced by the rapid advancements in technology that continuously raise the question: are the traditional approaches to surgical management always the best option? Indeed, by witnessing the conceptualization of CBT as a new, novel idea, further galvanized by the cumulative effects of various biomechanical, morphometric, and 
clinical evidence that contributed to the development of CBT, one is able to gain an appreciation for the importance of sustained vigilance and scientific curiosity in optimizing patient outcomes in surgery. A rough search for "CBT" in PubMed provides a visual depiction of the explosive rise in popularity of CBT (Figure 4), owing to its novel and innovative premise backed by a foundation of research made by many significant figures in the field. This historical note provides an in-depth overview of the timeline of CBT and the key milestones that contributed to its development. 


\section{REFERENCES}

1. Roy-Camille R, Saillant G, Mazel C. Internal fixation of the lumbar spine with pedicle screw plating. Clin Orthop Relat Res. 1986(203): 7-17.

2. Kabins MB, Weinstein JN. The History of Vertebral Screw and Pedicle Screw Fixation. The Iowa Orthopaedic Journal. 1991;11: 127-36.

3. An HS, Singh K, Vaccaro AR, et al. Biomechanical evaluation of contemporary posterior spinal internal fixation configurations in an unstable burst-fracture calf spine model: special references of hook configurations and pedicle screws. Spine (Phila $\mathrm{Pa}$ 1976). 2004;29(3): 257-62.

4. Kim YJ, Lenke LG, Cho SK, et al. Comparative analysis of pedicle screw versus hook instrumentation in posterior spinal fusion of adolescent idiopathic scoliosis. Spine (Phila Pa 1976). 2004;29(18): 2040-8.

5. Parker JW, Lane JR, Karaikovic EE, et al. Successful short-segment instrumentation and fusion for thoracolumbar spine fractures: a consecutive 41/2-year series. Spine (Phila Pa 1976). 2000;25(9): 1157-70.

6. Tian NF, Huang QS, Zhou P, et al. Pedicle screw insertion accuracy with different assisted methods: a systematic review and meta-analysis of comparative studies. Eur Spine J. 2011;20(6): 846-59.

7. Yazu M, Kin A, Kosaka R, et al. Efficacy of novel-concept pedicle screw fixation augmented with calcium phosphate cement in the osteoporotic spine. J Orthop Sci. 2005;10(1): 56-61.

8. Wilkes RA, Mackinnon JG, Thomas WG. Neurological deterioration after cement injection into a vertebral body. J Bone Joint Surg Br. 1994;76(1): 155. 
9. Wan S, Lei W, Wu Z, et al. Biomechanical and histological evaluation of an expandable pedicle screw in osteoporotic spine in sheep. Eur Spine J. 2010;19(12): 2122-9.

10. Santoni BG, Hynes RA, McGilvray KC, et al. Cortical bone trajectory for lumbar pedicle screws. Spine J. 2009;9(5): 366-73.

11. Buck JE. Direct repair of the defect in spondylolisthesis. Preliminary report. J Bone Joint Surg Br. 1970;52(3): 432-7.

12. Henderson ED. Results of the surgical treatment of spondylolisthesis. J Bone Joint Surg Am. 1966;48(4): 619-42.

13. Morscher E, Gerber B, Fasel J. Surgical treatment of spondylolisthesis by bone grafting and direct stabilization of spondylolysis by means of a hook screw. Arch Orthop Trauma Surg. 1984;103(3): 175-8.

14. Jeanneret B. Direct repair of spondylolysis. Acta Orthop Scand Suppl. 1993;251: 1115.

15. Bradford DS, Iza J. Repair of the defect in spondylolysis or minimal degrees of spondylolisthesis by segmental wire fixation and bone grafting. Spine (Phila Pa 1976). 1985;10(7): 673-9.

16. Mohammed N, Patra DP, Narayan V, et al. A comparison of the techniques of direct pars interarticularis repairs for spondylolysis and low-grade spondylolisthesis: a metaanalysis. Neurosurg Focus. 2018;44(1): E10-E.

17. Willett K, Hearn TC, Cuncins AV. Biomechanical testing of a new design for Schanz pedicle screws. J Orthop Trauma. 1993;7(4): 375-80.

18. Esses SI, Bednar DA. The spinal pedicle screw: techniques and systems. Orthop Rev. 1989;18(6): 676-82. 
19. Barber JW, Boden SD, Ganey T, et al. Biomechanical study of lumbar pedicle screws: does convergence affect axial pullout strength? J Spinal Disord. 1998;11(3): 215-20.

20. Zindrick MR, Wiltse LL, Doornik A, et al. Analysis of the morphometric characteristics of the thoracic and lumbar pedicles. Spine (Phila Pa 1976). 1987;12(2): $160-6$.

21. Cook SD, Salkeld SL, Whitecloud TS, et al. Biomechanical evaluation and preliminary clinical experience with an expansive pedicle screw design. J Spinal Disord. 2000;13(3): 230-6.

22. Dhawan A, Klemme WR, Polly DW. Thoracic Pedicle Screws : Comparison of Start Points and Trajectories. Spine (Phila Pa 1976). 2008;33(24): 2675-81.

23. Steel TR, Rust TM, Fairhall JM, et al. Monosegemental Pedicle Screw Fixation for Thoraco-Lumbar Burst Fracture. Orthopaedic Proceedings. 2004;86-B(SUPP_IV): $458-$

24. Mobbs RJ. The "medio-latero-superior trajectory technique": an alternative cortical trajectory for pedicle fixation. Orthop Surg. 2013;5(1): 56-9.

25. Abimanyi-Ochom J, Watts J, Sanders K. Osteoporosis Costing all Australians. A New Burden of Disease Analysis 2012-2022. 2013. p

26. Cook SD, Salkeld SL, Stanley T, et al. Biomechanical study of pedicle screw fixation in severely osteoporotic bone. Spine J. 2004;4(4): 402-8.

27. Zhang QH, Tan SH, Chou SM. Effects of bone materials on the screw pull-out strength in human spine. Med Eng Phys. 2006;28(8): 795-801.

28. Halvorson TL, Kelley LA, Thomas KA, et al. Effects of bone mineral density on pedicle screw fixation. Spine (Phila Pa 1976). 1994;19(21): 2415-20.

29. Wittenberg RH, Shea M, Swartz DE, et al. Importance of bone mineral density in instrumented spine fusions. Spine (Phila Pa 1976). 1991;16(6): 647-52. 
30. Hirano T, Hasegawa K, Takahashi HE, et al. Structural characteristics of the pedicle and its role in screw stability. Spine (Phila Pa 1976). 1997;22(21): 2504-9; discussion 10.

31. Mueller JU, Baldauf J, Marx S, et al. Cement leakage in pedicle screw augmentation: a prospective analysis of 98 patients and 474 augmented pedicle screws. J Neurosurg Spine. 2016;25(1): 103-9.

32. Matsukawa K, Yato $\mathrm{Y}$, Kato T, et al. In vivo analysis of insertional torque during pedicle screwing using cortical bone trajectory technique. Spine (Phila Pa 1976). 2014;39(4): E240-5.

33. Sterba W, Kim D-G, Fyhrie DP, et al. Biomechanical analysis of differing pedicle screw insertion angles. Clin Biomech (Bristol, Avon). 2007;22(4): 385-91.

34. Lehman RA, Jr., Polly DW, Jr., Kuklo TR, et al. Straight-forward versus anatomic trajectory technique of thoracic pedicle screw fixation: a biomechanical analysis. Spine (Phila Pa 1976). 2003;28(18): 2058-65.

35. Matsukawa K, Yato Y, Nemoto O, et al. Morphometric measurement of cortical bone trajectory for lumbar pedicle screw insertion using computed tomography. J Spinal Disord Tech. 2013;26(6): E248-53.

36. Zdeblick TA, Kunz DN, Cooke ME, et al. Pedicle screw pullout strength. Correlation with insertional torque. Spine (Phila Pa 1976). 1993;18(12): 1673-6.

37. Okuyama K, Abe E, Suzuki T, et al. Can insertional torque predict screw loosening and related failures? An in vivo study of pedicle screw fixation augmenting posterior lumbar interbody fusion. Spine (Phila Pa 1976). 2000;25(7): 858-64.

38. Matsukawa K, Yato Y, Hynes RA, et al. Comparison of Pedicle Screw Fixation Strength Among Different Transpedicular Trajectories: A Finite Element Study. Clin Spine Surg. 2017;30(7): 301-7. 
39. Perez-Orribo L, Kalb S, Reyes PM, et al. Biomechanics of lumbar cortical screw-rod fixation versus pedicle screw-rod fixation with and without interbody support. Spine (Phila Pa 1976). 2013;38(8): 635-41.

40. Calvert GC, Lawrence BD, Abtahi AM, et al. Cortical screws used to rescue failed lumbar pedicle screw construct: a biomechanical analysis. J Neurosurg Spine. 2015;22(2): 166-72.

41. Baluch DA, Patel AA, Lullo B, et al. Effect of physiological loads on cortical and traditional pedicle screw fixation. Spine (Phila Pa 1976). 2014;39(22): E1297-302.

42. Phan K, Ramachandran V, Tran TM, et al. Systematic review of cortical bone trajectory versus pedicle screw techniques for lumbosacral spine fusion. J Spine Surg. 2017;3(4): 679-88.

43. Phan K, Hogan J, Maharaj M, et al. Cortical Bone Trajectory for Lumbar Pedicle Screw Placement: A Review of Published Reports. Orthop Surg. 2015;7.

44. Ueno $\mathrm{M}$, Imura $\mathrm{T}$, Inoue $\mathrm{G}$, et al. Posterior corrective fusion using a double-trajectory technique (cortical bone trajectory combined with traditional trajectory) for degenerative lumbar scoliosis with osteoporosis: technical note. J Neurosurg Spine. 2013;19(5): 600-7.

45. Gonchar IMD, Kotani YMD, Matsumoto YMD. Cortical Bone Trajectory versus Percutaneous Pedicle Screw in Minimally Invasive Posterior Lumbar Fusion. The spine journal. 2014;14(11): S114-S5.

46. Iwatsuki K, Yoshimine T, Ohnishi Y, et al. Isthmus-guided cortical bone trajectory for pedicle screw insertion. Orthop Surg. 2014;6(3): 244-8.

47. Mizuno M, Kuraishi K, Umeda Y, et al. Midline lumbar fusion with cortical bone trajectory screw. Neurol Med Chir (Tokyo). 2014;54(9): 716-21. 
48. Okudaira $\mathrm{T}$, Konishi $\mathrm{H}$, Baba H, et al. Comparison study of lumbar interbody fusion with cortical bone trajectory screws versus conventional open posterior lumbar interbody fusion. Proceeding of SMISS Global Forum. 2014.

49. Rodriguez A, Neal MT, Liu A, et al. Novel placement of cortical bone trajectory screws in previously instrumented pedicles for adjacent-segment lumbar disease using CT image-guided navigation. Neurosurg Focus. 2014;36(3): E9.

50. Xuan J, Xie CL, Wu Y, et al. Cortical Bone Trajectory Screw Fixation in the Upper and Middle Thoracic Spine (T1-T8): An Anatomic and Radiographic Assessment. World Neurosurg. 2018;116: e1023-e31.

51. Lai Z, Shi SY, Fei J, et al. [Case-control study on cortical bone trajectory screw and pedicle screw internal fixation for the treatment of senile patients with lumbar tuberculosis]. Zhongguo Gu Shang. 2020;33(7): 636-42. chi.

52. Matsukawa K, Kato T, Mobbs R, et al. Combination of sacral-alar-iliac screw and cortical bone trajectory screw techniques for lumbosacral fixation: technical note. $\mathrm{J}$ Neurosurg Spine. 2020;33(2): 1-191.

53. Kim YJ, Bridwell KH, Lenke LG, et al. Pseudarthrosis in long adult spinal deformity instrumentation and fusion to the sacrum: prevalence and risk factor analysis of 144 cases. Spine (Phila Pa 1976). 2006;31(20): 2329-36.

54. Le XF, Shi Z, Wang QL, et al. Rate and Risk Factors of Superior Facet Joint Violation during Cortical Bone Trajectory Screw Placement: A Comparison of RobotAssisted Approach with a Conventional Technique. Orthop Surg. 2020;12(1): 133-40.

55. Khan A, Rho K, Mao JZ, et al. Comparing Cortical Bone Trajectories for Pedicle Screw Insertion using Robotic Guidance and Three-Dimensional Computed Tomography Navigation. World Neurosurg. 2020;141: e625-e32. 
56. Vicente AM, Ballensiefen W, Jönsson J-I. How personalised medicine will transform healthcare by 2030: the ICPerMed vision. J Transl Med. 2020;18(1): 180-.

57. Kim J, Rajadurai J, Choy WJ, et al. Three-Dimensional Patient-Specific Guides for Intraoperative Navigation for Cortical Screw Trajectory Pedicle Fixation. World Neurosurg. 2019;122: 674-9.

58. Cheng H, Chen BP, Soleas IM, et al. Prolonged Operative Duration Increases Risk of Surgical Site Infections: A Systematic Review. Surg Infect (Larchmt). 2017;18(6): 722-35.

59. Maruo K, Arizumi F, Kusuyama K, et al. Accuracy and safety of cortical bone trajectory screw placement by an inexperienced surgeon using 3D patient-specific guides for transforaminal lumbar interbody fusion. J Clin Neurosci. 2020;78: 147-52.

60. Marengo N, Matsukawa K, Monticelli M, et al. Cortical Bone Trajectory Screw Placement Accuracy with a Patient-Matched 3-Dimensional Printed Guide in Lumbar Spinal Surgery: A Clinical Study. World Neurosurg. 2019;130: e98-e104. 


\section{FIGURE LEGENDS}

Figure 1. A. Normal anatomy. B. Standard/traditional pedicle screw insertion C.

Percutaneous pedicle fixation. D. CBT/medio-lateral superior technique.

Figure 2. Insertional techniques. A. Standard trajectory. B. LMIT (latero-medial inferior). C. MLIT (medio-lateral inferior). D. MLST (medio-lateral superior).

Figure 3. Evolution of trajectory of the CBT/MLST technique. The purple screw represents the initial reported technique using shorter and narrower pedicle screws. The yellow screw represents the evolution of the technique with a more superior starting point on the posterior elements and a longer trajectory of the screw.

Figure 4. A historical overview of CBT overlapped on a timeline of PubMed results with the search terms ("cortical bone trajectory") and ("pedicle screws"). 


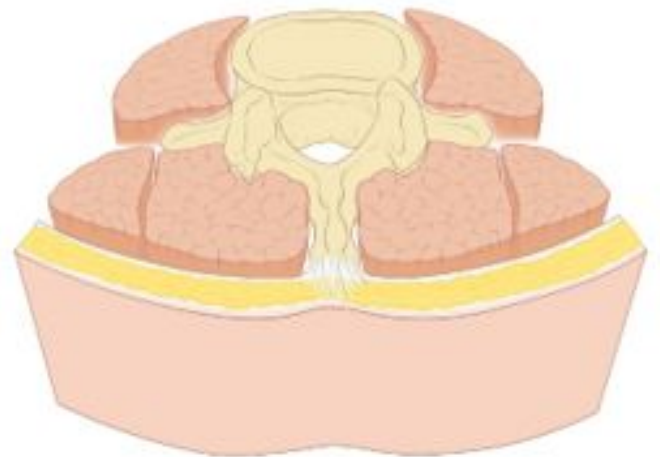

A. Normal

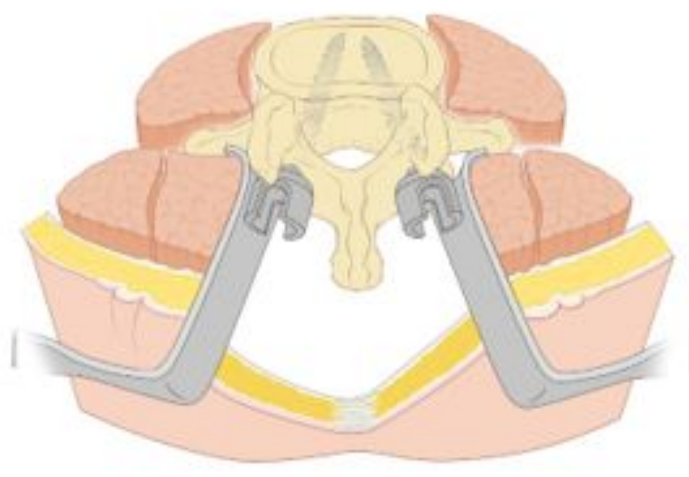

B. Standard

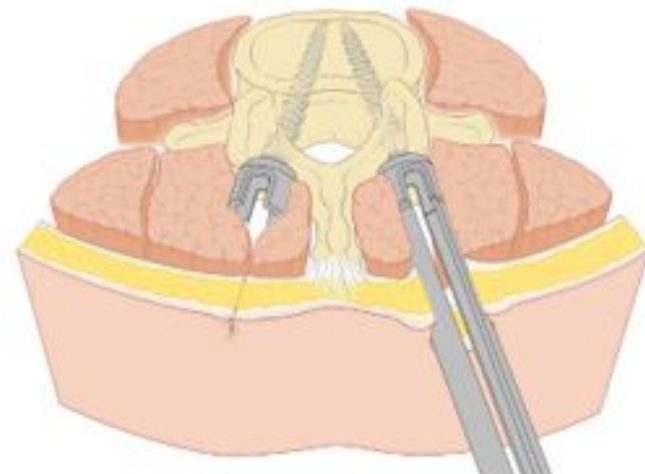

C. Percutaneous

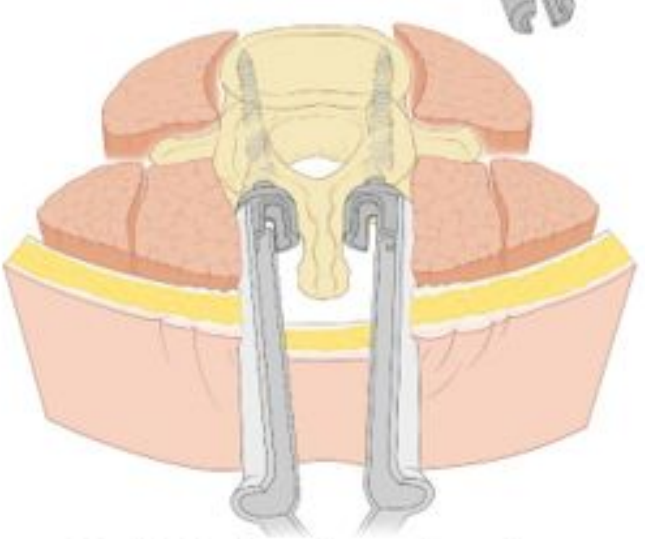

D. Medio-lateral superior trajectory

Figure 1 

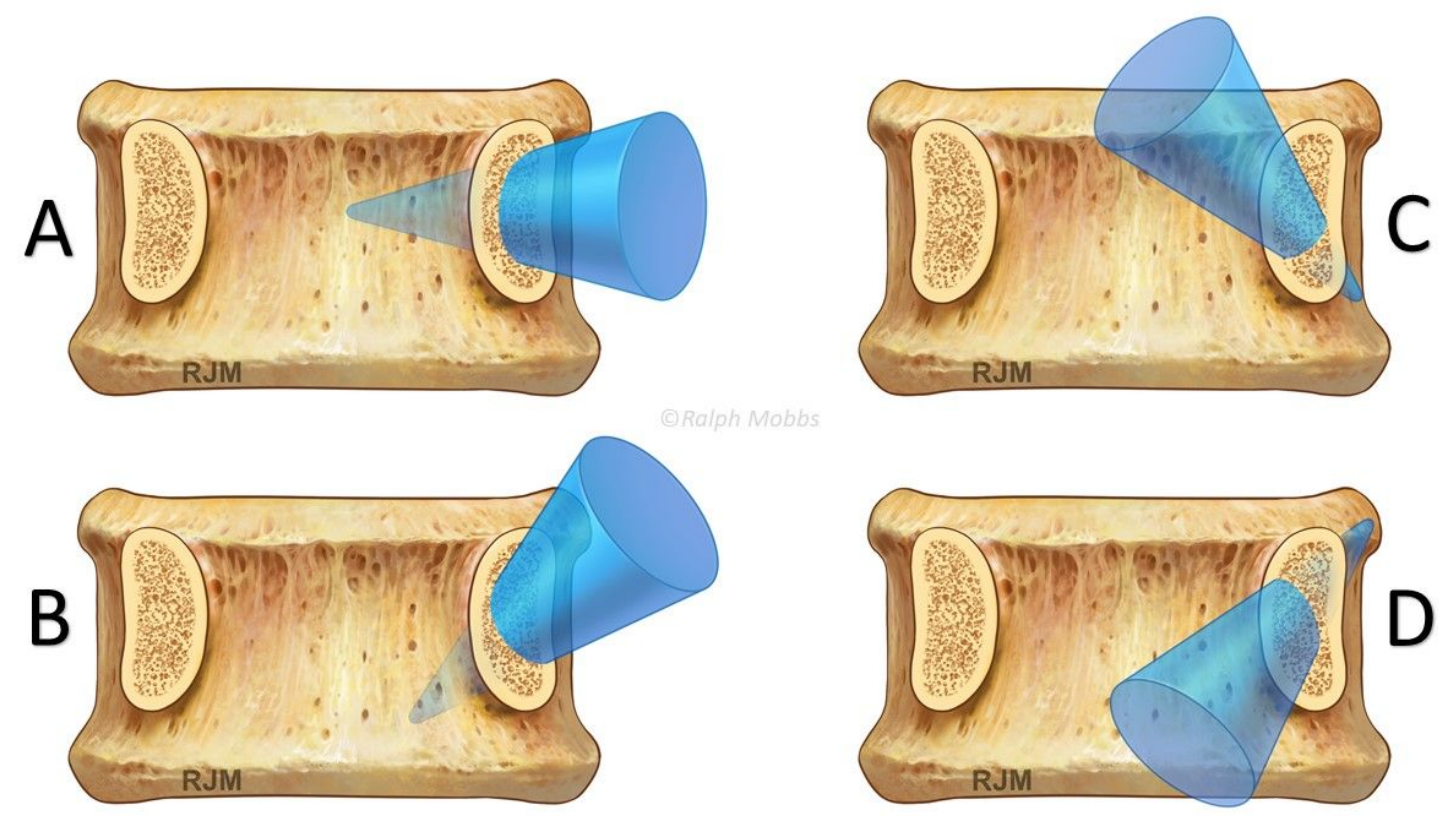

Figure 2 


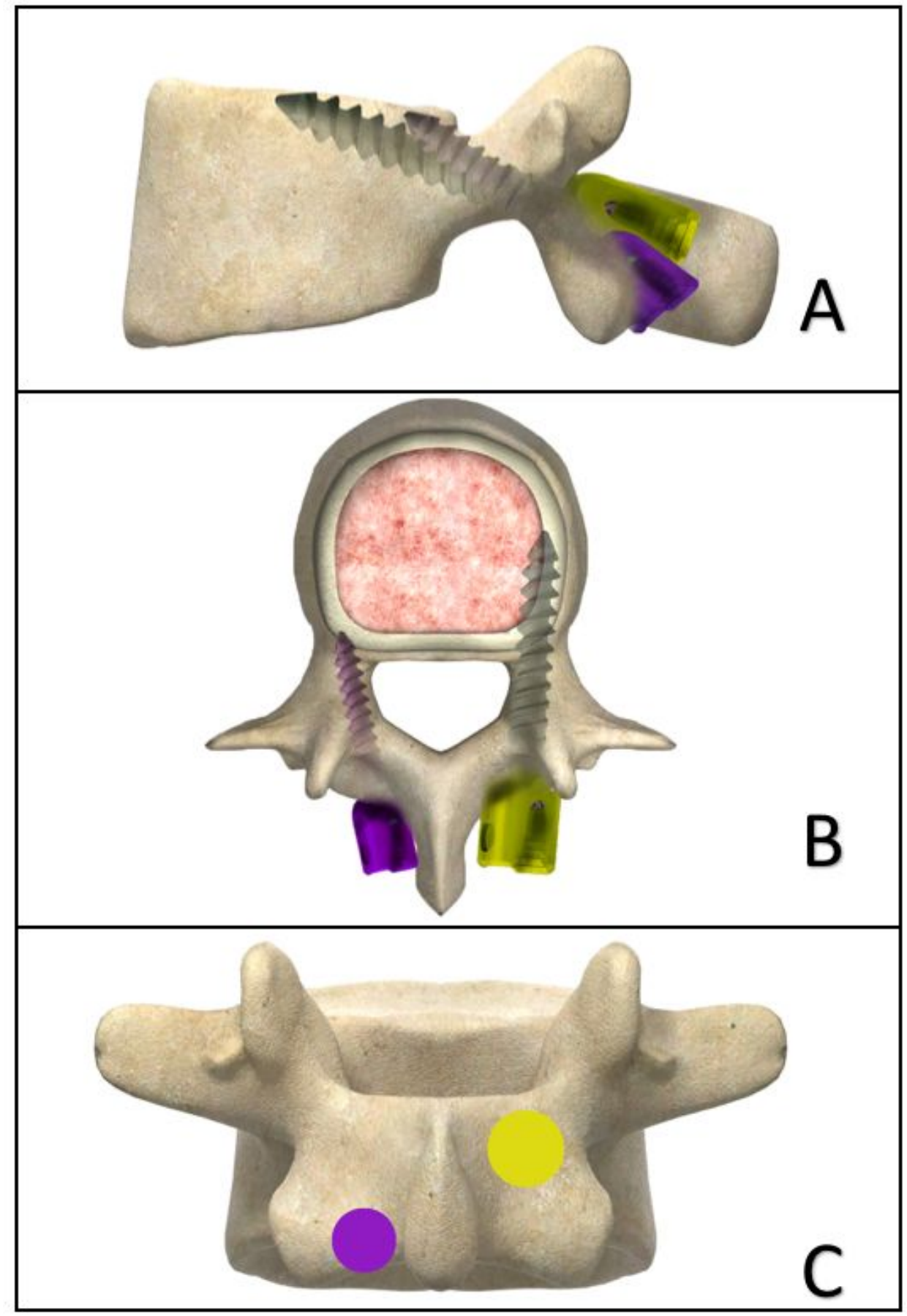

Figure 3 


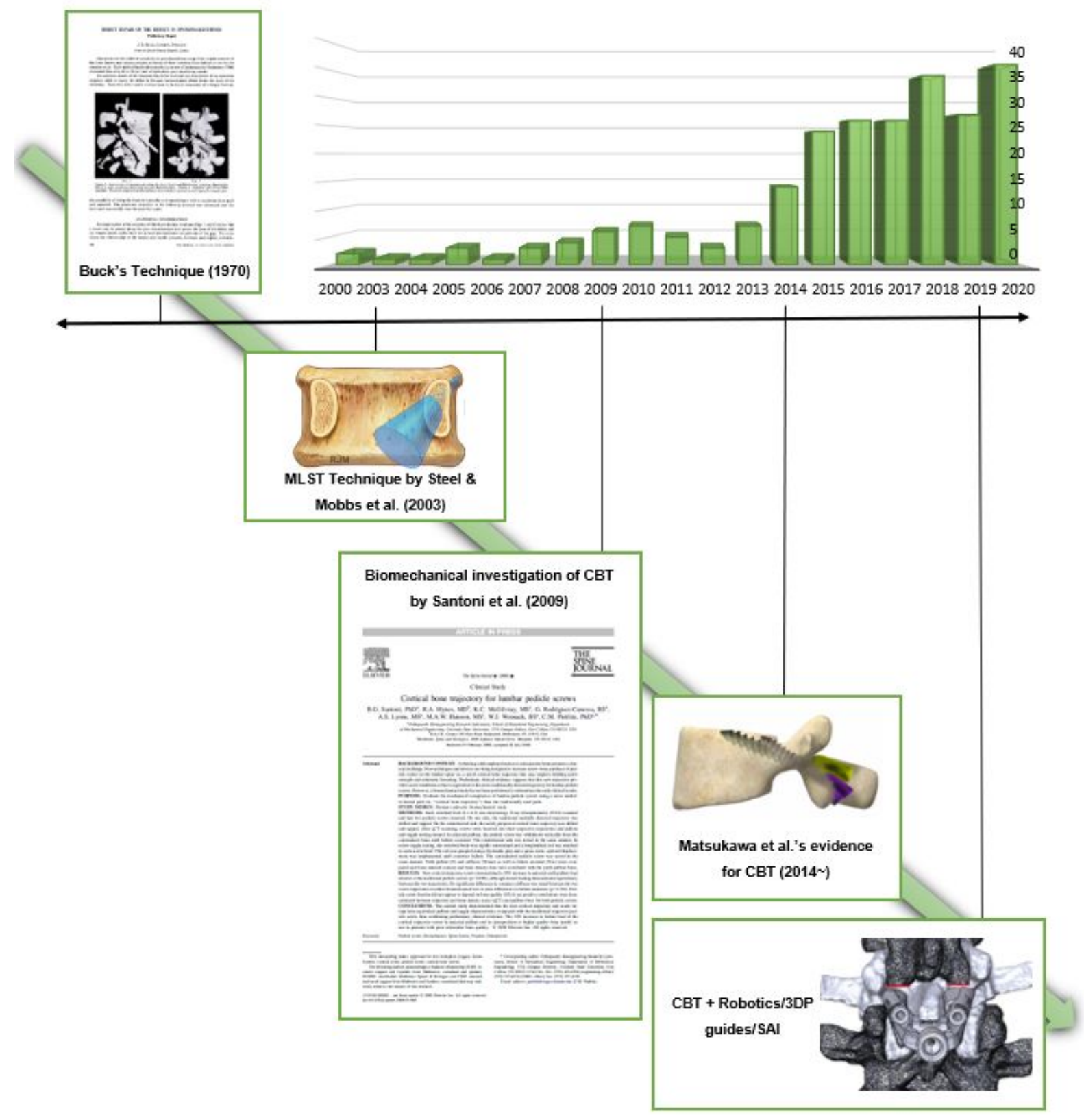

Figure 4 